\title{
Contact allergy in the population of patients with chronic inflammatory dermatoses and contact hypersensitivity to corticosteroids
}

\author{
Marek Kot ${ }^{1}$, Jarosław Bogaczewicz', Beata Kręcisz², Anna Woźniacka ${ }^{1}$ \\ 'Department of Dermatology and Venereology, Medical University of Lodz, Lodz, Poland \\ ${ }^{2}$ Faculty of Medicine and Health Science, Jan Kochanowski University, Kielce, Poland
}

Adv Dermatol Allergol 2017; XXXIV (3): 253-259 DOI: https://doi.org/10.5114/ada.2017.67848

\begin{abstract}
Introduction: Clinical studies indicate that contact allergy to glucocorticosteroids (GCS) is not rare and has been increasingly reported over the past decade. Among the risk factors for developing contact hypersensitivity to topical corticosteroids, chronic inflammatory skin diseases and polyvalent contact allergy seem to be most important. Aim: To present the structure of contact allergy in the population of patients with chronic inflammatory dermatoses (CID) and contact hypersensitivity to corticosteroids.

Material and methods: Twenty-seven patients with contact allergy to GCS and chronic inflammatory dermatoses were patch tested with 28 European Baseline Series allergens and 8 corticosteroid allergens. This study group consisted of 5 patients with atopic dermatitis (AD), 15 patients with contact eczema (CE) and 7 with chronic leg eczema (CLE). Nineteen (70.4\%) patients were females and 8 (29.6\%) were males.

Results: In the study group, the most sensitizing non-steroidal allergens were nickel sulfate (51.8\%), cobalt chloride (33.3\%) and balsam of Peru (29.6\%). The most sensitizing corticosteroid allergens were budesonide (77.8\%), betamethasone valerate and clobetasol propionate ( $55.5 \%$ each). A total of $77.8 \%$ of patients allergic to GCS also showed sensitivity to at least one non-steroidal allergen from the European Baseline Series.

Conclusions: The most important risk factors for developing contact allergy to corticosteroids appear to be chronic inflammatory dermatoses, long disease duration, extended on-and-off topical corticosteroid use, patients presenting two or more positive patch test results and polyvalent contact allergy to metal salts and to other non-steroidal haptens.
\end{abstract}

Key words: contact dermatitis, chronic dermatoses, allergy to corticosteroids.

\section{Introduction}

Topical corticosteroids have been used since the 1950s. However, a few years after its introduction, the first cases of contact allergy to glucocorticosteroids (GCS) were reported [1]. Paradoxically, these corticosteroids, whose anti-inflammatory and immunosuppressive properties made them suitable for use in the treatment of allergic diseases, also caused hypersensitivity reactions. These allergic reactions have been reported with oral, intramuscular, intraarticular, intravenous, topical and inhaled glucocorticoids, with Gell and Coombs scale type IV and I reactions being most commonly reported [2]. Systemic contact dermatitis to GCS could be presented as urticaria, exanthematous rash or purpura in pre-sensitized patients after the use of oral or inhaled steroids [3]. Studies have demonstrated that although GCS molecules themselves do not sensitize, their degradation products may do so. After conversion by epidermal esterases, steroid molecules form a highly reactive hapten called glyoxal (GLX), which gains the ability to induce a contact allergic reaction after being combined with a protein [4].

Contact sensitivity to GCS has been increasingly reported over the past decade [5], and it should be suspected if the patient has not responded to therapy with steroids, or the changes have worsened after using them. Among the risk factors for developing contact hypersensitivity to topical corticosteroids, chronic inflammatory skin diseases, patients presenting two or more positive

Address for correspondence: Marek Kot MD, Department of Dermatology and Venereology, Medical University of Lodz, pl. Hallera 1, 90-647 Lodz, Poland, phone: +48 4263930 92, e-mail: marek.kot@umed.lodz.pl Received: 11.09.2016, accepted: 13.11.2016. 
patch test results and polyvalent contact allergy to metal salts and to other non-steroidal haptens seem to be most important [6]. Contact allergy to glucocorticosteroids may affect $12.8 \%$ of patients with atopic dermatitis, $20 \%$ of patients with contact eczema and $40 \%$ of patients with chronic leg ulcers [7, 8]. Contact allergy to GCS is not easy to diagnose. To increase the detection of contact hypersensitivity to steroids, some authors advocate performing routine patch tests in patients experiencing chronic dermatoses with a long disease duration and long-term (on-and-off) topical steroid use [9].

\section{Aim}

This paper presents our clinical experience with patch testing to the European Standard Series and corticosteroid allergens in a specific population of patients with contact allergy to GCS and chronic dermatoses.

\section{Material and methods}

The clinical data and patch test results were reviewed of patients under the care of the Department of Dermatology and Venereology, Medical University of Lodz with chronic inflammatory dermatoses and contact allergy to GCS. The study group consisted of 27 patients in total: 5 patients with atopic dermatitis, 15 patients with contact eczema and 7 with chronic leg eczema. Diagnosis of atopic dermatitis (AD), contact eczema (CE) and chronic leg eczema (CLE) was set up on the basis of clinical signs, medical history and histological examination. Nineteen (70.4\%) patients were females and 8 (29.6\%) were males (age range: $23-82$ years, mean age: 48.9 years).

Before applying the patch tests, detailed interviews were carried out with the patients regarding the previous course of the disease and the treatment. Disease

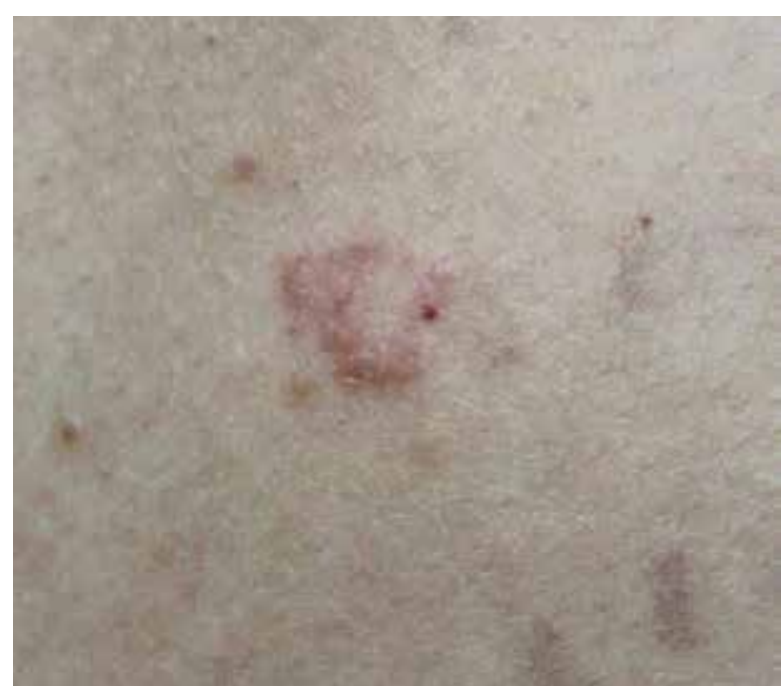

Figure 1. The ring effect with tixocortol pivalate duration was found to cover a period from 4 to 42 years, with an average of 10.6 years. The interviewer paid particular attention to the applicable topical corticosteroids. Time of using steroids topically (on and off) ranged from 4 months to 20 years, with a mean duration of 5.7 years. All patients had used topical steroids in their lifetime. Twenty-six (96.3\%) patients were using local therapy ointments containing corticosteroids from group D1, 23 (85.2\%) from group A, 11 (40.7\%) from group D2, 6 (22.2\%) from group B and 4 (14.8\%) were using topical corticosteroids from group C. No patient took corticosteroids, immunosuppressive drugs or antihistamines during the examination.

Only patients who had no treatment with antihistamines in the previous week, or in whom immunosuppressive drugs had been stopped in the previous 2 weeks were qualified for patch testing. Patients were patch tested with 28 European Baseline Series allergens and 8 corticosteroid allergens in different concentrations and in different media. Patch tests were read in all patients after 48, 72, 96, 120 and 168 hours since imposition. Irritant reactions were not taken into account in calculating the prevalence of allergy. In one case, a ring effect with tixocortol pivalate was observed (Figure 1).

\section{Results}

While $40.7 \%$ of the study group had experienced a disease term shorter than 5 years, 59.3\% had experienced a longer term. Similarly, while $59.3 \%$ of the group has undergone intermittent topical steroid therapy for less than 5 years, $40.7 \%$ of the patients has undergone more than 5 years of therapy. No patient demonstrated an allergy only to one hapten. Simultaneous hypersensitivity to 9, 12 and 13 allergens was observed in each case in $3.7 \%$ of patients, contact allergy to 4,6 and 14 haptens occurred in each case in 7.4\%, to 2 and 5 in $11.1 \%$ and to 3 haptens in $14.8 \%$ of cases. Simultaneous allergy to 8 haptens was reported in $29.6 \%$ of patients. A total of $77.8 \%$ of patients with hypersensitivity to GCS were also sensitized to non-steroidal haptens included in the European Baseline Series. In addition, 33.3\% of patients demonstrated an allergic reaction to one steroid allergen only. Simultaneous contact allergy to more than one steroid hapten was observed in $66.7 \%$ of cases. Simultaneous hypersensitivity to 2, 4 and 7 steroid haptens was observed in each case in $11.1 \%$ of patients, contact allergy to 3 and 8 haptens occurred in each case in $7.4 \%$, while contact hypersensitivity to 6 steroid allergens occurred in $18.5 \%$ of cases.

In the study group, the most common non-steroidal allergens giving positive results were nickel sulfate (51.8\%), cobalt chloride (33.3\%) and balsam of Peru (29.6\%) (Table 1). While the most frequently sensitizing non-steroidal haptens were nickel sulfate (63.1\%) and cobalt chloride (42.1\%) in the female population, the most 
Table 1. Prevalence of contact allergy to the European Baseline Series haptens in the population of patients with contact allergy to glucocorticosteroids

\begin{tabular}{|c|c|c|c|c|c|c|}
\hline \multirow[t]{3}{*}{ Haptens (concentration percentage and vehicle) } & \multicolumn{6}{|c|}{ Positive patch tests } \\
\hline & \multicolumn{2}{|c|}{ Female $(N=19)$} & \multicolumn{2}{|c|}{ Male $(N=8)$} & \multicolumn{2}{|c|}{ Total $(N=27)$} \\
\hline & $n$ & $\%$ & $n$ & $\%$ & $n$ & $\%$ \\
\hline Potassium dichromate (0.5\%, pet.) & 4 & 21 & 3 & 37.5 & 7 & 25.9 \\
\hline Phenylenediamine (1\%, pet.) & 0 & 0 & 0 & 0 & 0 & 0 \\
\hline Thiuram mix (1\%, pet.) & 0 & 0 & 0 & 0 & 0 & 0 \\
\hline Neomycin sulfate ( $20 \%$, pet.) & 2 & 10.5 & 0 & 0 & 2 & 7.4 \\
\hline Cobalt chloride ( $1 \%$, pet.) & 8 & 42.1 & 1 & 12.5 & 9 & 33.3 \\
\hline Benzocaine (5\%, pet.) & 1 & 5.3 & 2 & 25 & 3 & 11.1 \\
\hline Nickel sulfate ( $5 \%$, pet.) & 12 & 63.1 & 2 & 25 & 14 & 51.8 \\
\hline Clioquinol (5\%, pet.) & 1 & 5.3 & 0 & 0 & 1 & 3.7 \\
\hline Colophonium (20\%, pet.) & 2 & 10.5 & 2 & 25 & 4 & 14.8 \\
\hline Paraben mix (16\%, pet.) & 2 & 10.5 & 1 & 12.5 & 3 & 11.1 \\
\hline P-Phenylenediamine ( $0.1 \%$, pet.) & 0 & 0 & 0 & 0 & 0 & 0 \\
\hline Lanolin alcohol (30\%, pet.) & 1 & 5.3 & 0 & 0 & 1 & 3.7 \\
\hline Mercapto mix (2\%, pet.) & 0 & 0 & 0 & 0 & 0 & 0 \\
\hline Epoxy resin (1\%, pet.) & 0 & 0 & 0 & 0 & 0 & 0 \\
\hline Myroxylon pereirae resin ( $25 \%$, pet.) & 5 & 26.3 & 3 & 37.5 & 8 & 29.6 \\
\hline Para tertiary butylphenol formaldehyde resin (1\%, pet.) & 0 & 0 & 0 & 0 & 0 & 0 \\
\hline 2-Mercaptobenzothiazole (2\%, pet.) & 0 & 0 & 1 & 12.5 & 1 & 3.7 \\
\hline Formaldehyde (1\%, aq.) & 0 & 0 & 0 & 0 & 0 & 0 \\
\hline Fragrance mix I (8\%, pet.) & 1 & 5.3 & 1 & 12.5 & 2 & 7.4 \\
\hline Sesquiterpene lactone mix $(0.1 \%$, pet.) & 0 & 0 & 0 & 0 & 0 & 0 \\
\hline Quaternium-15 (1\%, pet.) & 0 & 0 & 0 & 0 & 0 & 0 \\
\hline 2-Methoxy-6-n-pentyl-4-benzoquinone ( $0.01 \%$, pet.) & 0 & 0 & 0 & 0 & 0 & 0 \\
\hline Methylisothiazolinone $(0.01 \%$, aq.) & 0 & 0 & 0 & 0 & 0 & 0 \\
\hline Budesonide $(0.01 \%$, pet.) & 13 & 68.4 & 5 & 62.5 & 18 & 66.7 \\
\hline Tixocortol-21-pivalate $(0.1 \%$, pet.) & 5 & 26.3 & 3 & 37.5 & 8 & 29.6 \\
\hline Metylodibromoglutaronitrile (0.5\%, pet.) & 3 & 15.8 & 0 & 0 & 3 & 11.1 \\
\hline Fragrance mix II (14\%, pet.) & 2 & 10.5 & 0 & 0 & 2 & 7.4 \\
\hline Lyral (5\%, pet.) & 0 & 0 & 0 & 0 & 0 & 0 \\
\hline
\end{tabular}

common non-steroidal allergens giving positive results in the male population were balsam of Peru and potassium dichromate $(37.5 \%$ each).

In the study population, the most common sensitizing steroidal allergens were budesonide (77.8\%), betamethasone valerate and clobetasol propionate $(55.5 \%$ each) (Table 2). The most common positive results in the female population were obtained with budesonide (73.7\%) and betamethasone valerate (47.4\%), while budesonide and clobetasol propionate (each 87.5\%) and valerate $(75 \%)$ most commonly elicited a positive response in the male population. In addition, $63 \%$ of patients with hypersensitivity to steroids were also allergic to metal salts. Contact allergy to metals was observed in $73.7 \%$ of females and $37.5 \%$ of males.

Corticosteroids included in the European Baseline Series (budesonide 0.01\% and tixocortol-21-pivalate) allowed $85.2 \%$ cases of steroid allergy to be detected. Allergy to budesonide was observed in $77.8 \%$ of patients: $71.4 \%$ of cases demonstrated a positive result at concentrations of $0.1 \%$ and $0.01 \%$, while $14.3 \%$ of cases returned a positive result at a concentration of $0.01 \%$ and $14.3 \%$ at a concentration of $0.1 \%$.

Contact allergy to tixocortol pivalate was diagnosed in $29.6 \%$ of all patients: $50 \%$ of these cases demonstrated an allergy to tixocortol pivalate alone, simultaneous 
Table 2. Prevalence of contact hypersensitivity to corticosteroid haptens in the population of patients with contact allergy to glucocorticosteroids

\begin{tabular}{lccccccc}
\hline Haptens (concentration percentage and vehicle) & Structural & \multicolumn{5}{c}{ Positive patch tests } \\
\cline { 3 - 8 } & group & & Female $(\boldsymbol{N}=19)$ & Male $(\boldsymbol{N}=8)$ & \multicolumn{2}{c}{ Total $(\boldsymbol{N}=27)$} \\
\cline { 3 - 8 } & & $n$ & $\%$ & $n$ & $\%$ & $n$ & $\%$ \\
\hline Budesonide (0.1\%, pet.) & $\mathrm{B}$ & 11 & 57.9 & 7 & 87.5 & 18 & 66.7 \\
\hline Betamethasone-17-valerate (0.1\%, pet.) & D1 & 9 & 47.4 & 6 & 75 & 15 & 55.5 \\
\hline Triamcinolone acetonide (1\%, pet.) & B & 7 & 36.8 & 3 & 37.5 & 10 & 37 \\
\hline Alclomethasone-17.21-dipropionate (1\%, pet.) & D1 & 1 & 5.3 & 1 & 12.5 & 2 & 7.4 \\
\hline Clobetasol-17-propionate (1\%, pet.) & D1 & 8 & 42.1 & 7 & 87.5 & 15 & 55.5 \\
\hline Dexamethasone-21-phosphate disodium salt (1\%, pet.) & C & 8 & 42.1 & 4 & 50 & 12 & 44.4 \\
\hline Hydrocortisone-17-butyrate (1\%, alk.) & D2 & 7 & 36.8 & 4 & 50 & 11 & 40.7 \\
\hline Hydrocortisone (1\%, pet.) & D2 & 8 & 42.1 & 3 & 37.5 & 11 & 40.7 \\
\hline Budesonide (0.01\%, pet.) & B & 13 & 68.4 & 5 & 62.5 & 18 & 66.7 \\
\hline Tixocortol-21-pivalate (0.1\%, pet.) & A & 5 & 26.3 & 3 & 37.5 & 8 & 29.6 \\
\hline
\end{tabular}

${ }^{*}$ Results of the European Baseline Series.

Table 3. Prevalence of contact allergy to the structural groups of steroids

\begin{tabular}{|c|c|c|c|c|c|c|}
\hline \multirow{3}{*}{$\begin{array}{l}\text { GCS - structural } \\
\text { groups }\end{array}$} & \multicolumn{6}{|c|}{ Positive patch tests } \\
\hline & \multicolumn{2}{|c|}{ Female $(N=19)$} & \multicolumn{2}{|c|}{ Male $(N=8)$} & \multicolumn{2}{|c|}{ Total $(N=27)$} \\
\hline & $n$ & $\%$ & $n$ & $\%$ & $n$ & $\%$ \\
\hline A & 5 & 26.3 & 2 & 25 & 7 & 25.9 \\
\hline B & 14 & 73.7 & 7 & 87.5 & 21 & 77.8 \\
\hline C & 9 & 47.4 & 4 & 50 & 13 & 48.1 \\
\hline D1 & 10 & 52.6 & 7 & 87.5 & 17 & 63 \\
\hline D2 & 10 & 52.6 & 5 & 62.5 & 15 & 55.5 \\
\hline
\end{tabular}

GCS - glucocorticosteroids.

Table 4. Prevalence of cross-reactions between structural groups of corticosteroids

\begin{tabular}{ccc}
\hline \multirow{2}{*}{$\begin{array}{c}\text { gCS }- \text { structural } \\
\text { groups }\end{array}$} & \multicolumn{2}{c}{ Prevalence of cross-reactions $(\boldsymbol{N}=\mathbf{2 7})$} \\
\cline { 2 - 3 } & 16 & $\%$ \\
\hline $\mathrm{BD}$ & 14 & 59.2 \\
\hline $\mathrm{BD} 1$ & 12 & 51.8 \\
\hline $\mathrm{BD} 2$ & 11 & 44.4 \\
\hline $\mathrm{D} 1 \mathrm{D} 2$ & 11 & 40.7 \\
\hline $\mathrm{BC}$ & 11 & 40.7 \\
\hline $\mathrm{CD}$ & 10 & 40.7 \\
\hline $\mathrm{CD} 1$ & 9 & 37 \\
\hline $\mathrm{CD} 2$ & 5 & 33.3 \\
\hline $\mathrm{AD}$ & 4 & 18.5 \\
\hline $\mathrm{AB}$ & 4 & 14.8 \\
\hline $\mathrm{AD} 2$ & 3 & 14.8 \\
\hline $\mathrm{AD} 1$ & 1 & 11.1 \\
\hline $\mathrm{AC}$ & & 3.7 \\
\hline
\end{tabular}

GCS - glucocorticosteroids. hypersensitivity to tixocortol pivalate and budesonide at concentrations of $0.01 \%$ and $0.1 \%$ was observed in $12.5 \%$, and simultaneous hypersensitivity to tixocortol pivalate and budesonide at concentrations of $0.1 \%$ and $0.01 \%$ was observed in $25 \%$.

Allergy to hydrocortisone butyrate was observed in $51.8 \%$ of all patients. In $57.1 \%$ of these cases, a positive result was observed for hydrocortisone butyrate in petrolatum and alcohol, 28.6\% demonstrated a positive result for hydrocortisone in petrolatum, and $14.3 \%$ demonstrated a positive result for hydrocortisone butyrate in alcohol. All patients who had previously returned positive results for patch testing for glucocorticoids, observed exacerbation of skin lesions or no improvement after using topical steroids.

In the study population, contact sensitivity to steroid group B occurred in $77.8 \%$ of patients, to group D1 in $63 \%$, to D2 in $55.5 \%$ of patients, to group C in $48.1 \%$ and to group $A$ in $25.9 \%$ of cases (Table 3 ). In the group of CID patients with contact hypersensitivity to steroids, most frequent cross-reactivity was observed between 
Table 5. Characteristics of contact allergy to metal salts in the population of patients with allergy to glucocorticosteroids

\begin{tabular}{|c|c|c|c|c|c|c|}
\hline \multirow[t]{3}{*}{ Haptens } & \multicolumn{6}{|c|}{ Positive patch tests } \\
\hline & \multicolumn{2}{|c|}{ Female $(N=19)$} & \multicolumn{2}{|c|}{ Male $(N=8)$} & \multicolumn{2}{|c|}{ Total $(N=27)$} \\
\hline & $n$ & $\%$ & $n$ & $\%$ & $n$ & $\%$ \\
\hline Ni only & 4 & 21 & 0 & 0 & 4 & 14.8 \\
\hline Co only & 0 & 0 & 0 & 0 & 0 & 0 \\
\hline Cr only & 1 & 5.3 & 1 & 12.5 & 2 & 7.4 \\
\hline $\mathrm{Ni} / \mathrm{Co}^{*}$ & 6 & 31.6 & 0 & 0 & 6 & 22.2 \\
\hline $\mathrm{Ni} / \mathrm{Cr}^{*}$ & 1 & 5.3 & 1 & 12.5 & 2 & 7.4 \\
\hline $\mathrm{Co} / \mathrm{Cr}^{*}$ & 1 & 5.3 & 0 & 0 & 1 & 3.7 \\
\hline $\mathrm{Ni} / \mathrm{Co} / \mathrm{Cr}^{*}$ & 1 & 5.3 & 1 & 12.5 & 2 & 7.4 \\
\hline Not present allergy to metals & 5 & 26.3 & 5 & 62.5 & 10 & 37 \\
\hline
\end{tabular}

$\mathrm{Ni}$ - nickel, Co-cobalt, $\mathrm{Cr}$ - chromate; *simultaneously.

the following structural groups of glucocorticosteroids: BD (59.2\%), BD1 (51.8\%) and BD2 (44.4\%) (Table 4).

Contact allergy to metal salts was found in $63 \%$ of patients: $73.7 \%$ of females and $37.5 \%$ of males. An allergy to one hapten only was noted in $22.2 \%$ of cases, to two allergens together in $33.3 \%$, and to three metal haptens simultaneously in $7.4 \%$. In the group aged under 40 years, hypersensitivity to metal salts was found in $81.8 \%$ of the patients $(88.9 \%$ of females and $50 \%$ of males), while in the population of over 40 years of age, contact allergy to metals occurred in $50 \%$ of patients (75\% of females and $33.3 \%$ of males). Simultaneous hypersensitivity to potassium dichromate and nickel sulfate was found in $7.4 \%$ of patients, allergy to cobalt chloride and potassium dichromate was found in 3.7\% of patients, allergy to cobalt chloride and nickel sulfate was found in $22.2 \%$ of patients, while simultaneous hypersensitivity to potassium dichromate, nickel sulfate and cobalt chloride occurred in $7.4 \%$ of patients (Table 5 ).

\section{Discussion}

Contact allergy to GCS has been recognized as a problem of clinical and therapeutic importance. The risk factors for the development of contact allergy to steroids are CIDs such as CE, AD, CLD, chronic leg ulcers (CLU) and stasis dermatitis (SD). Contact allergy to glucocorticosteroids may concern $12.8 \%$ of patients with AD, $20 \%$ of patients with CE and $40 \%$ of patients with CLU $[7,8]$. Risk factors for developing contact hypersensitivity to steroids include the presence of 2 or more positive patch test results and polyvalent contact sensitivities to metal salts and to other non-steroidal haptens [6]. The risk of contact allergy to corticosteroids increases with the duration of the disease and time of using topical steroids.

In our study, the mean disease duration was 10.6 years and the average duration of using topical GCS was 5.7 years. The most common sensitizing steroidal allergens were budesonide (77.8\%), betamethasone valerate and clobetasol propionate (55.5\% each). $77.8 \%$ of patients with hypersensitivity to GCS were also sensitized to non-steroidal haptens included in the European Baseline Series. No patient showed allergy to one hapten only. In the study group, the most common non-steroidal allergens giving positive results were nickel sulfate (51.8\%), cobalt chloride (33.3\%) and balsam of Peru (29.6\%).

Metal salts are the most frequent contact allergens in the world. Nickel allergy is most common among young women and with a positive history of pierced ears [10]. Isolated allergy to cobalt is very rare. Contact hypersensitivity to cobalt usually coexists with allergy to chromium in males and to nickel in females. Contact allergy to chrome is more common in men, older people and sufferers of occupational dermatitis [11]. In the present study, contact allergy to metal salts was found in 63\% of patients ( $73.7 \%$ of females and $37.5 \%$ of males). This allergy was to one hapten in $22.2 \%$ of cases, and to more than one in $40.7 \%$. Similar results were obtained by other authors.

In a group of 315 patients with contact allergy to steroids, $76 \%$ of females and $24 \%$ of males, mean age 48 years, Baeck et al. found most allergenic haptens to be budesonide (61\%), tixocortol pivalate (43\%), hydrocortisone butyrate (31\%) and prednisolone (22\%). In total, $27 \%$ of patients allergic to budesonide did not show hypersensitivity to tixocortol pivalate. Positive patch tests with two or more steroid haptens were achieved in $98.4 \%$ of patients. In cases with contact allergy to steroids, $84 \%$ also showed positive results with non-steroidal allergens. The most common non-steroidal haptens were fragrance mix (29\%), nickel (23\%), balsam of Peru (19\%), lanolin (17\%), colophony (15\%), neomycin (15\%), cobalt (14\%), p-phenylenediamine (13\%) and chromium (11\%) [12].

These results confirm that polyvalent contact hypersensitivity to non-steroidal allergens is a risk factor for allergy to steroids. A study by Gönül et al. on patients with contact eczema who did not respond to topical steroid therapy found that contact allergy to GCS was 
diagnosed in $22 \%$ of patients. The mean age of the cases was 33 years. Most positive results were obtained with the following steroids: tixocortol pivalate (14.6\%), budesonide, triamcinolone acetonide, alclometasone propionate (4.9\% each). Polyvalent allergy was diagnosed in $44.4 \%$ of patients with hypersensitivity to GCS [13].

In a study conducted by Davis et al. on 1188 patients who were suspected to have an allergy to steroids, 127 patch test results showed a positive reaction to one hapten, and 56 turned out to be simultaneously hypersensitive to more than one. Contact allergy to GCS was demonstrated in $10.69 \%$ of patients with contact eczema. Tixocortol pivalate (5.03\%) and budesonide 0.1\% (3.07\%) were found to be the most common sensitivities [14]. The results of our present study show that the most common risk factor for contact allergy to steroids is polyvalent allergy to metal salts, but also to other nonsteroidal haptens. Similarly, Reitamo et al. suggest that patients suffering from chronic dermatoses and those who experienced two or more positive tests with nonsteroidal haptens have an increased risk of allergy to corticosteroids [15].

Budesonide is considered a diagnostic allergen for the B and D corticosteroid groups. Due to the fact that budesonide gives very frequent cross-reactions, it is regarded as one of the best markers of contact allergy to GCS [16]. Tixocortol pivalate is a group A steroid and it is regarded as the best marker of allergy to hydrocortisone [17]. The authors showed that tixocortol pivalate and budesonide $0.01 \%$ allow even more than $90 \%$ of patients with contact hypersensitivity to GCS to be diagnosed [18]. In our research, corticosteroids included in the European Baseline Series allowed 85.2\% cases of allergy to GCS to be detected.

There are different opinions about the concentrations which should be used for corticosteroid allergen patch tests. Some authors consider that a high corticosteroid concentration increases allergen penetration and despite the strong anti-inflammatory effect, allows the demonstration of hypersensitivity, whereas other researchers suggest that the lower concentration of the substance allows a hypersensitivity reaction to be obtained more quickly. In a study conducted by Chowdhury et al. on a population with contact eczema, positive patch test results with tixocortol pivalate in a concentration of $1 \%$ was found in $1.74 \%$ of patients, while patch tests with this substance in a concentration of $0.1 \%$ were positive in $0.95 \%$. Positive results of patch tests with $0.1 \%$ budesonide was present in $0.62 \%$ of patients, while with this substance in a concentration of $0.01 \%$ were positive in $0.43 \%$ of cases [19].

In our research, contact sensitivity to group B steroids was found to occur in $77.8 \%$ of patients and to group D1 steroids in $63 \%$ of cases. Most frequent cross-reactivity was observed between the BD (59.2\%), BD1 (51.8\%) and BD2 (44.4\%) groups. Results of the study confirm that budesonide is the best marker of allergy to steroids because of the common cross-reactions. In a study recorded by Baeck et al., most common positive results were obtained with the following structural groups of steroids: B (29\%), A (28\%), C (17\%), D1 (16\%), and D2 (10\%) [12]. In the study by Davis et al., positive results with the following groups were the most frequent: $A(5.72 \%), B$ and D (4.8\% each), D1 (3.54\%), D2 (2, 13\%) and C (1.1\%) [14]. In a study by Gönül and Gül, cross-reactions between the following groups of steroids were the most common: BD, BD1, BC and BA (22.2\% each) [13].

\section{Conclusions}

Our findings confirm that the risk of allergy to corticosteroids increases with the duration of the disease and time of using topical steroids. The most important risk factors for steroid allergy are chronic inflammatory dermatoses and simultaneous polyvalent allergy to nonsteroidal haptens, especially metal salts. The present study also confirms that budesonide, due to its structure, is one of the best diagnostic allergens for contact allergy to GCS.

\section{Acknowledgments}

This study was supported by a grant for scientific purposes No. 502-03/1-152-01/502-14-254 from the Medical University of Lodz, Poland.

\section{Conflict of interest}

The authors declare no conflict of interest.

\section{References}

1. Burckhardt W. Klinische und Therapeutische Kurzberichte. Hautarzt 1959; 10: 42-3.

2. Venturini M, Lobera T, del Pozo MD, et al. Immediate hypersensitivity to corticosteroids. I Investig Allergol Clin Immunol 2006; 16: 51-6.

3. Mckenna DB, Murphy GM. Contact allergy to topical corticosteroids and systemic allergy to prednisolone. Contact Dermatitis 1998; 38: 121-2.

4. Wilkinson SM, Jones SF. Corticosteroid usage and binding to arginine: determinants of corticosteroid hypersensitivity. $\mathrm{Br}$ J Dermatol 1996; 135: 225-30.

5. Reduta T, Bacharewicz J, Pawłoś A. Patch test results in patients with allergic contact dermatitis in the Podlasie region. Postep Derm Alergol 2013; 30: 350-7.

6. Dooms-Gossens A, Degreef H, Mariën KJC, et al. Contact allergy to corticosteroids: a frequent missed diagnosis? J Am Acad Dermatol 1989; 21: 538-43.

7. Żmudzińska M, Czarnecka-Operacz M, Silny W. Contact allergy to glucocorticosteroids in patients with chronic venous leg ulcers, atopic dermatitis and contact allergy. Acta Dermatovenerol Croat 2008; 16: 72-8.

8. Kot M, Bogaczewicz J, Kręcisz B, et al. Contact hypersensitivity to haptens of the European standard series and cortico- 
steroid series in the population of adolescents and adults with atopic dermatitis. Dermatitis 2014; 25: 72-6.

9. Browne F, Wilkinson SM. Effective prescribing in steroid allergy: controversies and cross-reactions. Clin Dermatol 2011; 29: 287-94.

10. Jensen CS, Lisby S, Baadsgaard O, et al. Decrease in nickel sensitization in a Danish schoolgirl population with ears pierced after implementation of a nickel exposure regulation. Br J Dermatol 2002; 146: 636-42.

11. Kieć-Świerczyńska M. Allergy to chromate, cobalt and nickel in Lodz 1977-1988. Contact Dermatitis 1990; 22: 229-31.

12. Baeck M, Chemelle JA, Terreux R, et al. Delayed hypersensitivity to corticosteroids in a series of 315 patients: clinical data and patch test results. Contact Dermatitis 2009; 61: 163-75.

13. Gönül M, Gül U. Detection of contact hypersensitivity to corticosteroids in allergic contact dermatitis patients who do not respond to topical corticosteroids. Contact Dermatitis 2005; 53: 67-70.

14. Davis MD, el-Azhary RA, Farmer SA. Results of patch testing to a corticosteroids series a retrospective review of 1188 patients during 6 years at Mayo Clinic. J Am Acad Dermatol 2007; 56: 921-7.

15. Reitamo S, Lauerma Al, Stubb S, et al. Delayed hypersensitivity to topical corticosteroids. J Am Acad Dermatol 1986; 14: 582-9.

16. Matura M, Goosens A. Contact allergy to corticosteroids. Allergy 2000; 55: 698-704.

17. Wilkinson SM, English JSC. Hydrocortisone sensitivity: an investigation into the nature of the allergen. Contact Dermatitis 1991; 25: 171-81.

18. Seukeran DC, Wilkinson SM, Beck MH. Patch testing to detect corticosteroid allergy: Is it adequate? Contact Dermatitis 1997; 36: 127-30.

19. Chowdhury MMU, Statham BN, Sansom JE, et al. Patch testing for corticosteroid allergy with low and high concentrations of tixocortol pivalate and budesonide. Contact Dermatitis 2002; 46: 311-2. 